\title{
Stem cell therapy for cardiac disease
}

\author{
Harold S. Bernstein ${ }^{1}$ and Deepak Srivastava ${ }^{1}$
}

Congenital heart disease occurs in 1\% of liveborn infants, making it the most common birth defect worldwide. Many of these children develop heart failure. In addition, both genetic and acquired forms of dilated cardiomyopathy are a significant source of heart failure in the pediatric population. Heart failure occurs when the myocardium is unable to meet the body's metabolic demands. Unlike some organs, the heart has limited, if any, capacity for repair after injury. Heart transplantation remains the ultimate approach to treating heart failure, but this is costly and excludes patients who are poor candidates for transplantation given their comorbidities, or for whom a donor organ is unavailable. Stem cell therapy represents the first realistic strategy for reversing the effects of what has until now been considered terminal heart damage. We will discuss potential sources of cardiac-specific stem cells, including mesenchymal, resident cardiac, embryonic, and induced pluripotent stem cells. We will consider efforts to enhance cardiac stem cell engraftment and survival in damaged myocardium, the incorporation of cardiac stem cells into tissue patches, and techniques for creating bioartificial myocardial tissue as well as whole organs. Finally, we will review progress being made in assessing functional improvement in animals and humans after cellular transplant.

M ore than 19 million children and adults in the United States and Europe suffer with heart failure, resulting in $\sim 230,000$ deaths annually at a cost of $>\$ 140$ billion/y $(1,2)$. This includes the 2.5 million children born each year worldwide with congenital heart disease (3), many of whom eventually develop heart failure. Heart failure occurs when damaged myocardium becomes unable to meet the body's metabolic demands. Unlike some organs, the heart has a severely limited, if any, capacity for repair after injury.

Currently, heart transplantation remains the ultimate approach to treating end-stage heart failure, but this therapy is invasive, costly, and excludes patients who are not candidates for transplantation given their comorbidities. Most importantly, there are not enough organs for transplanting the increasing number of patients with end-stage heart failure, including children with complex congenital heart disease who are surviving well into adulthood. New, accessible therapies are needed to treat the millions of patients with debilitating heart failure worldwide. Myocardial engineering, including stem cell transplantation, may represent the first realistic strategy for reversing the effects of what has until now been considered terminal damage to the heart. We review the potential sources of cardiac-specific stem cells and their incorporation into tissue patches, as well as the progress made in assessing functional improvement in transplanted animals and human patients.

\section{CELL SOURCES}

\section{Mesenchymal Stem/Stromal Cells}

Mesenchymal stem/stromal cells (MSCs) reside in the bone marrow stroma and can differentiate into osteoblasts, chondrocytes, and adipocytes $(4,5)$. In addition, MSCs differentiate in vitro into spontaneously beating cardiomyocytes $(\mathrm{CMs})$ after exposure to the demethylating agent 5-azacytidine (6). Because of their cardiomyogenic potential, MSCs have been transplanted in animal models of myocardial infarction (MI). Collectively, these studies have demonstrated improved leftventricular function, reduced infarct scar size, attenuated leftventricular remodeling, greater vascular density, and increased survival following transplantation (7). Besides transdifferentiating into CMs, MSCs are relatively easy to grow and can be expanded in culture to cell numbers required for transplantation (8). Another advantage of MSCs for the repair of damaged myocardium is their ability to suppress immune rejection and curb the inflammatory response. Despite expressing major histocompatibility complex class I and low levels of class II antigens as well as Fas ligand, MSCs fail to elicit an alloreactive lymphocyte response when added to mixed lymphocyte cultures (9).

MSC transplantation has been tested in the clinic. In a randomized, placebo-controlled study, patients with acute MI received either intracoronary injections of autologous MSCs or saline (10). Evaluation of treated patients as compared with a placebo group and with that of pretransplantation after injury, the MSC-treated group showed increased wall movement velocity at the infarct site, left-ventricular ejection fraction, and end-systolic pressure to end-systolic volume ratio at 3-mo posttherapy. Cardiac mechanical and electrical properties were also significantly improved. Osiris Therapeutics completed a phase I clinical trial to assess the safety and preliminary efficacy of 
intravenous allogeneic adult human MSCs (11). Although MSC infusion proved safe in patients, with no ectopic tissue formation, the trial results also showed improved outcomes for MSC-treated patients with respect to left-ventricular function, cardiac arrhythmias, pulmonary function, and symptomatic global assessment. Whether MSCs transdifferentiate into new CMs remains controversial (12), and so the mechanisms for MSC-mediated functional benefit remain to be fully elucidated. However, several companies are now conducting phase II clinical trials to evaluate "off-the-shelf" MSC therapy in patients with acute MI and congestive heart failure.

\section{Resident Cardiac Stem Cells}

Since the 1960s, studies of CM proliferation in rodents had indicated that the adult mammalian heart was a terminally differentiated, postmitotic organ without the capacity for cellular regeneration (13). Over the past $10 \mathrm{y}$, however, several findings have challenged this view. Observation of cell division, telomerase activity, telomere shortening, and CM apoptosis have provided evidence of CM turnover in adult human hearts (14-17). In addition, pockets of mitotically active cells in hypertrophic myocardium and hearts of patients with end-stage heart failure have been described (18). Using a genetic fate-mapping approach, Hsieh et al. showed that whereas adult CMs are not replaced in the uninjured heart during normal aging up to $1 \mathrm{y}$ in the mouse, they are refreshed after MI or pressure overload (19). Recently, Porrello et al. showed that the hearts of 1-d-old neonatal mice can regenerate after partial surgical resection, but this capacity is lost by $7 \mathrm{~d}$ of age (20). The most compelling evidence for $\mathrm{CM}$ renewal in maintaining cardiac homeostasis comes from a study in which the amount of ${ }^{14} \mathrm{C}$ (generated from above-ground nuclear testing between 1955 and 1963, before the implementation of the Limited Nuclear Test Ban Treaty) integrated into the DNA of human myocardial cells was used to date the birth of myocardial cells (21). Carbon dating indicated postnatal cell turnover that declines with age: about $1 \%$ per year at age 25 to $0.45 \%$ at 75 . This provided strong evidence not only for low-level turnover of CMs in the adult heart but for the idea that adult myocardial tissue is capable of incorporating new muscle cells to preserve tissue mass and function.

c-Kit and stem cell antigen-1 as markers of cardiac progenitors. Detection of the $\mathrm{Y}$ chromosome in undifferentiated cells and differentiated CMs found in female donor hearts transplanted into male patients not only supports the concept of cardiac chimerism in humans but also suggests the existence of cardiac progenitor cells that give rise to new CMs (22). This putative stem cell population was positive for surface antigens c-Kit/ CD117 and/or multidrug resistance-like protein 1, or stem cell antigen-1 (Sca-1). Cardiac stem cells have been identified and isolated by others based on the expression of these markers $(17,23)$. In the presence of 5 -azacytidine or oxytocin, Sca-1+ cells are reported to exhibit spontaneous beating, express cardiac-specific genes, and, when injected intravenously into mice following MI, home to the infarct site, differentiate, and fuse with host myocardium (23). Likewise, multipotent c-Kit ${ }^{+}$ cells reconstitute the injured myocardium by forming new blood vessels and myocytes, comprising as much as $70 \%$ of the ventricle in some reports $(24,25)$. The structural and functional regeneration of infarcted myocardium occurred independently of cell fusion. Remarkably, Bearzi et al. obtained similar results after isolating human $\mathrm{c}-\mathrm{Kit}^{+}$cells from myocardial tissue of patients who underwent cardiac surgery, expanding them in culture, and injecting them into the infarcted myocardium of immunodeficient mice and immunosuppressed rats (26).

Side population cells. Side population (SP) cells, known for their ability to efflux vital dyes such as Hoechst 33342 , were initially discovered as hematopoietic stem cells (27). ATP-dependent transporters, including multidrug resistance-like protein 1 and ATP-binding cassette subfamily G member 2 (ABCG2), are believed to mediate dye exclusion, and both ABCG2 and multidrug resistance-like protein 1 have been cited as molecular determinants of cardiac SP cells in the adult myocardium $(28,29)$. Further characterization shows that cardiac SP cells widely express Sca-1 and CD31 but are largely negative for the hematopoietic markers CD45 and CD34, cellular adhesion marker CD44, and c-Kit (30). Moreover, functional differentiation and maturation of cardiac SP cells are also restricted to the Sca- $1^{+} / \mathrm{CD} 31^{-}$subpopulation. Direct injection of heartderived Sca- $1^{+} / \mathrm{CD} 31^{-}$cells into the peri-infarct region immediately following $\mathrm{MI}$ in mice limits left-ventricular remodeling, attenuates contractile dysfunction, and improves myocardial energy metabolism (31). The transplanted Sca- $1^{+} / \mathrm{CD} 31^{-}$cells promoted neo-angiogenesis and underwent in vivo differentiation into CMs and endothelial cells.

Cardiospheres. Cardiospheres present another potential source of endogenous cardiac stem cells. Cardiosphere cells are derived from cultured explants of mouse hearts and human atrial or ventricular biopsy samples following gentle enzymatic digestion (32). They are able to migrate over the adherent portion of the explanted tissues. When collected and cultured, they form clonal, multicellular clusters capable of self-renewal as well as differentiation into CMs and vascular cells. Immunophenotypic characterization shows that newly formed cardiospheres express stem cell markers CD34, c-Kit, and Sca-1, and endothelial markers CD31 and kinase insertion domain receptor/vascular endothelial growth factor receptor 2/Flk-1. Recently, Ye et al. have shown that the Sca- $1^{+}$subpopulation of cardiosphere cells preferentially include Isl-1-expressing precursors that give rise to second heart field structures (33). Since their description, alternative approaches and more efficient methods to generate cardiospheres have been developed $(34,35)$. Of particular note, the yield of cardiosphere cells from injured hearts is greater than from uninjured hearts, and cardiospheres are easily isolated and expanded from "middle aged" hearts (33), supporting their feasibility in autologous cell transplantation. Clinical studies in animal models of MI have shown that cardiosphere injection into infarcted mouse and pig hearts preserves ventricular function, improves hemodynamic 
indices, produces less adverse remodeling, and reduces infarct size $(33,34,36-38)$.

Despite displaying partial overlap in marker expression, the origins and exact lineage relationships among these various adult cardiac stem cells remain unknown. In fact, the validity of these cell populations as resident cardiac stem or progenitor cells has been questioned. Pouly et al. reported that c-Kit ${ }^{+}$cells harvested from either human right-ventricular endomyocardium or right-atrial appendage lack other stem cell markers such as multidrug resistance-like protein 1 , and coexpress $\mathrm{CD} 45$, suggesting they are of hematopoietic origin (39). In addition, c-Kit ${ }^{+}$ cells stained positive for the mast cell lineage marker tryptase, implying that these cells may not be CM precursors but rather mast cells. Studies in which Sca-1 was used to identify adult stem cells from human hearts were based on immunoreactivity to nonhuman Sca-1 antibodies. To date, a human homologue of Sca-1 has not been found (40), although the preponderance of studies that have identified cells based on the expression of "human Sca-1" make an immunoreactive analogue likely. There have also been some discrepant findings with regard to cardiospheres. Studies by several groups have demonstrated that some explant migrating cells do not differentiate into functional CMs or yield any significant physiological benefit in the infarcted mouse heart (41-43). Notwithstanding, the clinical utility of cardiospheres is currently being tested in phase I clinical trials in patients with MI (44).

\section{Human Embryonic Stem Cells}

Human embryonic stem cells (hESCs) grow and divide indefinitely while maintaining the potential to develop into derivatives of all three embryonic germ layers. Under appropriate culture conditions, a small fraction of hESCs (5-15\%) spontaneously differentiate into CMs with structural and functional properties characteristic of endogenous CMs (45). To improve yield of hESC-derived CMs for cell therapy, current efforts are focused on directing the differentiation of hESCs into the cardiac lineage (46). Innovative enrichment, purification, and selection strategies have been developed to guide cardiac differentiation to relatively pure homogeneity (Figure 1).

Defined culture media have been developed to direct CM differentiation from hESCs. Coculture studies with mouse END2 cells have identified prostaglandin $\mathrm{I}_{2}$ as an inducer of CM differentiation (47). Similarly, 5-azacytidine treatment during hESC differentiation has been shown to enhance CM differentiation, suggesting that DNA demethylation is a key factor in directing tissue-specific differentiation (48). Exposure to SB203580, a small-molecule inhibitor of $\mathrm{p} 38^{\mathrm{MAPK}}$, has also been observed to improve the efficiency of CM differentiation from hESCs $(49,50)$ and has implicated $\mathrm{p} 38^{\mathrm{MAPK}}$ in the regulation of the ectoderm-mesoendoderm switch during early ESC differentiation (50). By mimicking the signaling environment of the early mouse embryo, Yang et al. established a three-stage protocol that supports cardiac development at high frequency in differentiating hESC cultures (51). This protocol exposes human embryoid bodies (hEBs) to a combination of activin A, bone morphogenetic protein 4 , and basic fibroblast growth factor (bFGF) during the first $4 \mathrm{~d}$ of differentiation to induce primitive-streak formation, representing the onset of gastrulation. Between days 4 and 8 , the differentiating hEBs are treated with vascular endothelial growth factor and Dickkopf homolog 1 to induce the development of cardiac mesoderm and further maturation.

Advances in understanding the genetic and epigenetic regulation of CM differentiation have also suggested potential new approaches to producing stem cell-derived CMs for therapy. The small, regulatory RNAs, microRNA (miR)-1 and miR133 , are specifically expressed in the mouse heart, and their targeted deletion or knockdown results in dysregulation of cardiac morphogenesis, electrical conduction, cell cycle, and cardiac hypertrophy $(52,53)$. Recently, Ivey et al. showed that miR-1 and miR-133 regulate the differentiation of mouse embryonic stem cells (mESCs) and hESCs into the cardiac lineage (54). Lentiviral induction of either miR-1 or miR-133 expression in mESCs enhanced early mesoderm differentiation and repressed development of endoderm and neuroectoderm. However, further differentiation revealed a predominant role for miR-1 in promoting the differentiation of $\mathrm{mESC}$ - or hESCderived mesoderm into cardiac and skeletal muscle cells. More recently, Wong et al. have shown that miR-125b similarly controls early CM specification through its effects on the pluripotency factor LIN28 (55). Whereas miRNAs direct cell lineage

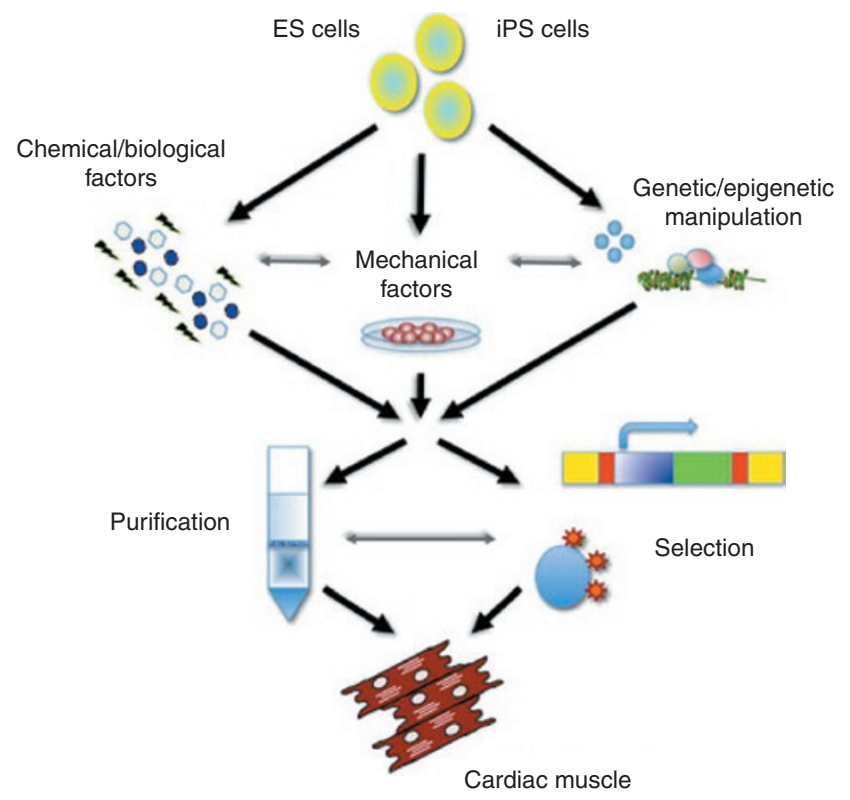

Figure 1. Approaches to preparing embryonic stem (ES) cell- and induced pluripotent stem (iPS) cell-derived cardiomyocytes (CMs) for tissue repair. Methods for directing differentiation of pluripotent stem cells to CMs have focused on chemical (e.g., 5-azacytidine, p38 ${ }^{\text {MAPK }}$ inhibitors, PGI2) and biological (e.g., activin A, bone morphogenetic protein, basic fibroblast growth factor, vascular endothelial growth factor, Dickkopf homolog 1) factors, genetic (e.g., miRNAs) and epigenetic (e.g., miRNAs, chromatin remodeling) manipulation, and mechanical factors (e.g., hydrodynamics, surface tension). In transplantation experiments, these approaches have been complemented by purification methods that take advantage of the biochemical properties of CMs (e.g., Percoll density centrifugation, mitochondrial content), and selection strategies that rely on the expression of cardiac-specific genes (e.g., reporter lines, molecular beacons) and surface markers. 
determination by controlling protein dosage, epigenetic regulation through chromatin remodeling has been shown to control cell fate as well. Takeuchi et al. identified a minimal set of factors necessary to execute the cardiac transcriptional program (56). Baf60c, a cardiac-enriched subunit of the Swi/ Snf-like barrier-to-autointegration (BAF) chromatin remodeling complex, in combination with cardiac transcription factors guanine-adenine-thymine-adenine (GATA) motif binding zinc finger transcription factor-4 (Gata4) and T-box transcription factor-5 (Tbx5), was able to induce cardiac differentiation in mouse embryos when ectopically expressed (56).

In combination with directed differentiation strategies, the use of genetic selection has the potential to address issues of $\mathrm{CM}$ homogeneity and isolation of cardiac progenitors. Several laboratories have developed transgenic reporter hESC lines to derive pure CM populations. This relies on a cardiac-restricted promoter to drive the expression of a reporter gene or selectable marker. Huber et al. used lentiviral vectors to produce stable hESC lines in which enhanced green fluorescent protein (eGFP) was expressed under control of the cardiac-specific human MLC2v promoter (57). Xu et al. generated stable hESC lines using a reporter consisting of the cardiac-specific mouse $\alpha$-myosin heavy chain promoter driving expression of the neomycin resistance gene (58). Kita-Matsuo et al. designed a set of lentiviral vectors to generate multiple stable hESC lines with eGFP and mCherry reporters or with puromycin resistance downstream of the mouse a-myosin heavy chain promoter (59). Recently, Ritner et al. generated a cardiac-specific hESC reporter line using a lentiviral construct consisting of a fragment of the mouse a-myosin heavy chain promoter upstream of eGFP. The specific promoter fragment allowed the identification and analysis of early cardiac progenitors expressing NKX2-5 before the onset of cardiac troponin $\mathrm{T}$ or chamberspecific myosin light chain (MLC) expression (60).

Collectively, fluorescence-activated cell sorting or antibiotic selection of these lines has yielded $85-99 \%$ pure CMs or cardiac progenitors. eGFP-expressing cells derived from the ventricular MLC2 (MLC2v) transgenic line formed stable intracardiac cell grafts following transplantation (57). Injection of neomycin resistance-selected hEBs into the hind limb muscles of SCID mice resulted in no teratoma formation after $23 \mathrm{wk}$ (58). Contractile forces in puromycin resistance-selected CMs were similar to those generated by rat neonatal ventricular CMs (59). Whereas isolation of hESC-derived CMs from these lines was based on positive selection, Anderson et al. implemented a negative selection strategy to deplete undifferentiated, proliferating hESCs from cultures of hESC-derived CMs (61). Their transgenic hESC line utilized a Herpes thymidine kinase/ganciclovir suicide gene system under the control of a constitutive phosphoglycerate kinase promoter. An important caveat of this approach, however, is that other nonproliferating cell types remained in the culture while proliferating CMs were depleted. CMs produced with this line still required purification, and the excluded proliferating progenitors may yet be proven to be of benefit for transplantation.

As an alternative to genetically modified hESC lines for tracking and isolating $\mathrm{CMs}$ and progenitors, King et al. adapted dual-fluorescence resonance energy transfer "molecular beacon" technology for transient, real-time detection of gene expression during hESC differentiation (62). Molecular beacons are single-stranded oligonucleotide probes that have been employed to assay gene expression in vitro, as in realtime PCR, and in vivo using microscopy. These investigators showed that appropriately designed, dual-fluorescence resonance energy transfer molecular beacon pairs can identify the expression of specific mRNAs and facilitate the collection of specific $\mathrm{hESC}$ populations by fluorescence-activated cell sorting, while leaving the hESC genome intact (62).

Transplantation with hESC-derived CMs in animal models of MI has yielded promising, albeit modest, results. Although the methods for generating CMs and monitoring engraftment, as well as the number of transplanted CMs, varied between studies, hESC-derived CM transplantation has led to measurable benefit (63-65). Overall, CM transplantation preserved leftventricular function, attenuated post-MI wall thinning, and even partially remuscularized the infarct region. Despite these encouraging results, the cardiac-specific benefits determined from in vivo engraftment studies appear transient. Van Laake et al. reported that although cardiac function was improved 4 wk after MI, the functional benefit was no longer significant at $12 \mathrm{wk}$, even after tripling the number of transplanted CMs in a repeat study (63). These results question the utility of fully differentiated CMs for cardiac repair. The integration of hESCderived CMs into existing muscle may be hampered by their limited developmental plasticity, whereas partially differentiated cardiac progenitors may retain the plasticity needed for extensive engraftment (60). In addition, the electrophysiological differences between human and mouse CMs, for example, the significant difference in baseline beating rates, might affect integration of human cells into mouse myocardium, limiting the conclusions that might be drawn from these studies.

Another limitation of hESC-derived CMs is that the allogeneic transplanted cells may be subject to immune rejection. hESCs appear to have a lower immunostimulatory potential as compared with adult cells. DNA microarray data of undifferentiated and differentiated hESCs indicate that almost half of the upregulated immunoregulatory genes in hematopoietic cells, lymphoid organs, and other tissues are not similarly expressed in hESCs, implying that hESCs are immunologically immature (66). This observation suggests that immunosuppressive regimens for hESC-based therapeutics may not need to be as rigorous as those for conventional organ transplantation (66). Nevertheless, transplanted hESC-derived CMs may be susceptible to immune rejection to some degree.

\section{Induced Pluripotent Stem Cells}

The advent of induced pluripotent stem cell (iPSC) technology $(67,68)$ offers a possible solution to immune rejection. This technology entails reprogramming terminally differentiated adult human fibroblasts to pluripotent stem cells through ectopic expression of four pluripotency factors: OCT4, SOX2, c-MYC, and KLF4. Reprogrammed cells exhibit many features characteristic of hESCs, including morphology, cell surface 
antigens, gene expression, telomerase activity, and proliferation rates, and have the capacity to differentiate into cells of all three germ layers, including CMs $(68,69)$. Because adult fibroblasts can be obtained directly from the patient, it has been assumed that patient-specific, iPSC-derived CMs could be transplanted without immune rejection. However, a recent study showed that isogenic iPSCs may still elicit an immune response (70). Another obstacle to iPSC-based therapy is the time needed to derive patient-specific cells. Using currently available protocols, iPSCs cannot be used under urgent circumstances, such as in the setting of acute MI or rapidly progressive heart failure. Although the prospect of iPSC banking to provide "off-the-shelf" therapy may be useful in genetically homogeneous populations $(71,72)$, this approach may not be practical in more diverse communities (73).

It is also important to recognize that iPSCs have typically been generated using viral vectors, which carry the risk of potentially harmful genomic integration. Indeed, some established iPSC lines are genetically unstable, exhibiting largescale genomic rearrangements, copy-number variations, and abnormal karyotype even at early passage $(74,75)$. Although most alternative methods for reprogramming are significantly less efficient as compared with viral integration $(76,77)$, several more efficient, nonintegrating technologies have recently been described $(78,79)$. Furthermore, in some iPSC lines reprogrammed using nonintegrating viral methods, high levels of mutational changes were still observed (74). Recent studies have also revealed that iPSCs contain epigenetic features that indicate either incomplete or aberrant reprogramming. In particular, iPSC DNA methylation patterns are frequently reminiscent of the somatic cell of origin (80), suggesting that iPSCs may be incompletely reprogrammed to the naive pluripotent state seen in hESCs. It is unclear whether the observed genetic instability and epigenetic imprinting accrues during reprogramming or was present in the somatic cell of origin. Nevertheless, patient-specific iPSCs derived from somatic cells of older patients may be more likely to contain genomic mutations and disadvantageous epigenetic programs. Thus, the safety and efficacy of therapeutic iPSCs as currently derived remain to be tested.

\section{Induced CMs}

A recent study provides another promising approach to bypassing immune rejection. Ieda et al. identified a minimal set of transcription factors to reprogram postnatal cardiac fibroblasts into functional CMs (81). They showed that Gata4, myocyte enhancer factor-2c (Mef2c), and Tbx 5 were sufficient for CM induction. Induced CMs expressed cardiac genes, exhibited calcium oscillations, and possessed action potentials resembling those of adult ventricular CMs. Some induced CMs reprogrammed fully to display spontaneous contractile activity. In vivo, cardiac fibroblasts transduced with Gata4, Mef2c, and Tbx 5 transdifferentiated into CMs within 2 wk after injection into immunosuppressed mouse hearts. This suggests that endogenous cardiac fibroblasts provide a pool of cells that could be transdifferentiated in vivo to regenerate heart muscle.

\section{TISSUE PATCHES}

To improve the survival and engraftment of transplanted cells, tissue engineering efforts have focused on in vitro cultivation systems in which cells are grown on preformed scaffolds. These afford matrix and culture parameters suitable for generating three-dimensional contractile and vascularized cardiac patches that resemble native cardiac muscle. Zimmermann et al. developed a technique to create engineered heart tissue whereby neonatal rat CMs were combined with liquid collagen I and Matrigel in circular molds subjected to mechanical strain (82). Grafts implanted post-MI in rats demonstrated structural and electrical integration with the host myocardium as well as improved diastolic and systolic ventricular function as compared with controls. Caspi et al. constructed highly porous, multicellular scaffolds composed of poly-L-lactic acid and polylactic-glycolic acid in which hESC-derived CMs, human endothelial cells, and embryonic fibroblasts were cultured (83). The three cell-based culture strategy generated human cardiac tissue that was vascularized and contracted spontaneously and synchronously with gap junctions mediating impulse conduction. Transplantation of the engineered cardiac tissue to healthy rat hearts resulted in the formation of stable tissue grafts and functional vascularization (84).

Bioreactors provide a way to control environmental conditions for constructing functional cardiac tissue and are designed to recapitulate the microenvironmental conditions within native cardiac muscle. Neonatal CMs within cardiac tissue patches cultivated under pulsatile fluid flow exhibited highly ordered sarcomeres, intercalated discs, and better contractile properties than those cultivated in nonpulsatile medium flow $(85,86)$. Application of electrical stimulation during cultivation of neonatal rat cardiac constructs enhanced ultrastructural differentiation and augmented contractile behavior as compared with nonstimulated constructs $(87,88)$. Using the rat omentum as a natural bioreactor to prevascularize neonatal rat cardiac patches, Dvir et al. showed that omentum-generated patches coupled structurally and electrically with the host myocardium $4 \mathrm{wk}$ after implantation and attenuated ventricular remodeling and dysfunction (89).

Decellularization of tissues to create native scaffolds is another approach that has been taken to reconstitute whole tissues and organs. Using detergent-based coronary perfusion to decellularize cadaveric rat hearts, Ott et al. produced a whole-heart scaffold with intact geometry, vasculature, and extracellular matrix (90). They then repopulated the decellularized heart with cardiac and endothelial cells and mounted the recellularized heart in a bioreactor. By supplying oxygen and electrical stimulation within the bioreactor, the organ displayed pumping capacity within several days. This decellularization/recellularization technique holds tremendous promise for the generation of bioartificial myocardial tissue, as well as whole organs for transplantation.

Beyond the use of conventional biodegradable scaffolds, Shimizu et al. engineered cell sheets using thermoresponsive cell culture dishes to detach intact sheets and then stacked them three-dimensionally to create thick cardiac constructs (91). The temperature-sensitive culture surfaces were covalently grafted with poly $(\mathrm{N}$-isopropylacrylamide) upon which 
neonatal rat CMs were grown. On reducing the temperature below $32^{\circ} \mathrm{C}$, confluent cells spontaneously lifted up as a single contiguous sheet with cell-cell junctions and extracellular matrix-deposited adhesive proteins preserved. In vitro, layered cardiac sheets pulsed simultaneously, and when implanted subcutaneously into nude rats, displayed spontaneous macroscopic beating, synchronized electrical potentials, neovascularization, and cardiac structures including sarcomeres, desmosomes, and gap junctions. Transplantation of three-dimensional cardiac sheets onto the epicardial surface of injured rat hearts resulted in functional integration of electrical signals between the cardiac graft and host heart without any observed arrhythmias (92).

\section{FUNCTIONAL ASSESSMENT IN ANIMAL MODELS}

\section{Hemodynamic Assessment}

Although some tissues and organs require that transplanted cells perform specific metabolic tasks, it is essential that cells transplanted to the heart coordinate both mechanically and electrically with native tissue. Testing the efficacy of cell-based therapies in various animal models of MI has created a need for accurate assessment of cardiovascular variables in the intact animal. In addition to tissue measurements of infarct size, vascularity, cellular proliferation, and CM apoptosis postmortem, changes in left-ventricular structure and function can be measured in vivo by a variety of techniques, of which serial echocardiography has been employed in the majority of studies. Left-ventricular end-systolic volume, end-diastolic volume, end-systolic diameter, and end-diastolic diameter, as well as anterior and posterior wall thickness during systole and diastole can be obtained with two-dimensional and M-mode echocardiography (65). These measurements allow for calculation of left-ventricular ejection volume, fractional shortening, and mass. Three-dimensional imaging using cardiac magnetic resonance or multidetector computerized tomography permits similar measurements but with a higher degree of precision and accuracy due to improved temporal and spatial resolution that also offers detailed examination of changes in tissue morphology $(42,93)$.

Other indices for quantifying global and regional leftventricular function can be obtained with Doppler tissue imaging, which measures blood flow velocities and strain rates, both of which have been shown to correlate with the maximum rate of rise of left-ventricular pressure, or $\mathrm{dP} / \mathrm{dt}_{\text {max }}$, an indicator of myocardial contractility (94). Some studies have used more invasive procedures such as pressure-volume loop analysis to directly measure hemodynamic parameters $(38,95)$. Leftventricular volume and pressure are recorded from a specially designed catheter with a pressure sensor and conductance electrodes for simultaneous pressure-volume measurements. End-systolic and end-diastolic volumes, heart rate, maximum pressure, and contractile and relaxation parameters can be obtained. Sonomicrometry has also been utilized to determine wall thickness, wall motion, segment shortening, end-systolic and end-diastolic pressure-volume relationships, stroke work, stroke volume, and ejection fraction (96). This technique involves implanting small piezo-electric transducer crystals in the infarct region of the heart, allowing the distances between the transducers to be measured continuously through ultrasound waves transmitted and received by the crystals.

\section{Electrophysiological Assessment}

Ventricular tachyarrhythmias and sudden cardiac death caused the suspension of clinical trials using autologous skeletal myoblasts transplanted into the myocardium of MI patients (97). This underscored the importance of electrical integration of transplanted cells into the recipient myocardium. Thus, a fundamental operating principle for cell transplantation is the requirement for electrical coupling with the host myocardium. In vitro and ex vivo, electrophysiological assessment has included patch clamp, multielectrode array, and optical mapping analyses. Patch clamp technique has been used to characterize action potentials from CMs derived from hESCs in vitro. Electrical recordings of dissociated hESC-derived CMs have revealed fetal ventricular, atrial, and pacemaker action potentials (98). hESC-derived cardiac action potentials were shown to be sensitive to the L-type calcium channel blocker verapamil, implicating the presence of L-type calcium channels; moreover, the L-type calcium channels were responsive to adrenergic stimuli (98). More recently, as much as one-third of hESC-derived CMs have been shown to possess a mature electrical phenotype, as determined by patch clamp analysis (99).

Multielectrode array mapping has been employed to acquire electrophysiological data. This technique consists of an array of embedded, substrate-integrated contact electrodes that allow simultaneous recording of extracellular field potentials from all electrodes over extended periods of time. The detected field potentials from the attached cells can be correlated to the shape and duration of the underlying action potential. Using multielectrode arrays to assess electrical conduction of hESCderived CMs, Satin et al. detected functional sodium channels that were sensitive to the sodium channel blocker tetrodotoxin (100). Ritner et al. used multielectrode arrays to show that hESCs give rise to a heterogeneous combination of chamberspecific cell types (60).

Optical mapping uses voltage-sensitive dyes to record action potentials ex vivo. Voltage-sensitive dyes bind with high affinity to the cell membrane and, when excited, emit light in direct proportion to the transmembrane voltage, thereby producing an optical signal that mimics an action potential. Optical mapping not only maps membrane depolarization but also the repolarization process. Verheule and coworkers utilized highresolution optical mapping to study atrial conduction in canine models of chronic atrial dilatation and chronic rapid atrial pacing to determine whether the underlying mechanisms of atrial fibrillation inducibility are similar (101). Based on the conduction profiles obtained, they found that the underlying substrate for atrial fibrillation in the chronic atrial dilatation model is distinctly different from that of the chronic rapid atrial pacing model. The same group used optical mapping to evaluate the electrophysiological effects of transplanted hESC-derived $\mathrm{CMs}$ on injured rat myocardium (102). High-resolution 
analysis demonstrated a slight conduction delay at the site of CM grafting.

The capacity of transplanted CMs to electrocouple with the host myocardium is often examined by postmortem immunohistochemical analysis, usually through connexin 43 immunohistochemistry to demonstrate that the transplanted cells form gap junctions within the infarcted region. Besides identification of proteins involved in establishing electrocellular connections, additional evidence is clearly warranted to demonstrate electromechanical stabilization between donor cells and host myocardium. One study used pigs as a large animal model of atrioventricular heart block to show functional electrical integration. Injection of hESC-derived CMs into the left ventricle of pig hearts with atrioventricular block resulted in successful pacing of the heart, manifested by the presence of a new ectopic ventricular rhythm measured by body surface electrocardiography and catheter-based electroanatomical mapping (103). In another study, hESC-derived CMs were injected subepicardially into the left-ventricular anterior wall of guinea pigs. After atrioventricular nodal cryoablation to suppress the intrinsic heart rhythm, ex vivo optical mapping of control cryoablated hearts exhibited complete electrical silence, whereas transplanted hearts propagated spontaneous action potentials from the site of injection (104).

\section{FUTURE PERSPECTIVES}

The past decade has improved our knowledge of stem cell biology and the development of the cardiovascular system. However, a more profound understanding of cardiac myogenesis will be required for the development of advanced stem cell therapeutics to repair or regenerate damaged myocardium. The future will likely include (i) further investigation to delineate the human CM lineage tree; (ii) methods to isolate specific cardiac progenitor pools or specialized CM subtypes; (iii) strategies to ensure survival of transplanted cells, their functional integration with the host myocardium, and circumvention of immune rejection; (iv) development of technologies to accurately assess integration; (v) determination of parameters that optimize engraftment, such as delivery method, timing of transplantation post-MI, and cell preparations; and (vi) large-animal models of heart failure that closely resemble human cardiovascular physiology and disease for assessing cell engraftment, host immune response, and myocardial function.

\section{ACKNOWLEDGMENTS}

We apologize to colleagues whose work could not be cited due to space limitations. We thank the members of our laboratories for helpful discussion.

\section{STATEMENT OF FINANCIAL SUPPORT}

H.S.B. is supported by grants from the National Institutes of Health, the California Institute for Regenerative Medicine, and the Muscular Dystrophy Association. D.S. is supported by grants from the National Institutes of Health/ National Heart, Lung, and Blood Institute and the California Institute for Regenerative Medicine.

\section{REFERENCES}

1. Lloyd-Jones D, Adams R, Carnethon M, et al.; American Heart Association Statistics Committee and Stroke Statistics Subcommittee. Heart disease and stroke statistics-2009 update: a report from the American Heart
Association Statistics Committee and Stroke Statistics Subcommittee. Circulation 2009;119:480-6.

2. Hogg K, Swedberg K, McMurray J. Heart failure with preserved left ventricular systolic function; epidemiology, clinical characteristics, and prognosis. J Am Coll Cardiol 2004;43:317-27.

3. Hoffman JI, Kaplan S. The incidence of congenital heart disease. J Am Coll Cardiol 2002;39:1890-900.

4. Jiang Y, Jahagirdar BN, Reinhardt RL, et al. Pluripotency of mesenchymal stem cells derived from adult marrow. Nature 2002;418:41-9.

5. Pittenger MF, Mackay AM, Beck SC, et al. Multilineage potential of adult human mesenchymal stem cells. Science 1999;284:143-7.

6. Makino S, Fukuda K, Miyoshi S, et al. Cardiomyocytes can be generated from marrow stromal cells in vitro. J Clin Invest 1999;103:697-705.

7. Boyle AJ, McNiece IK, Hare JM. Mesenchymal stem cell therapy for cardiac repair. Methods Mol Biol 2010;660:65-84.

8. Alhadlaq A, Mao JJ. Mesenchymal stem cells: isolation and therapeutics. Stem Cells Dev 2004;13:436-48.

9. Le Blanc K, Tammik C, Rosendahl K, Zetterberg E, Ringdén O. HLA expression and immunologic properties of differentiated and undifferentiated mesenchymal stem cells. Exp Hematol 2003;31:890-6.

10. Chen SL, Fang WW, Ye F, et al. Effect on left ventricular function of intracoronary transplantation of autologous bone marrow mesenchymal stem cell in patients with acute myocardial infarction. Am J Cardiol 2004;94:92-5.

11. Hare JM, Traverse JH, Henry TD, et al. A randomized, double-blind, placebo-controlled, dose-escalation study of intravenous adult human mesenchymal stem cells (prochymal) after acute myocardial infarction. J Am Coll Cardiol 2009;54:2277-86.

12. Rose RA, Keating A, Backx PH. Do mesenchymal stromal cells transdifferentiate into functional cardiomyocytes? Circ Res 2008;103:e120.

13. Soonpaa MH, Kim KK, Pajak L, Franklin M, Field LJ. Cardiomyocyte DNA synthesis and binucleation during murine development. Am J Physiol 1996;271(5 Pt 2):H2183-9.

14. Leri A, Barlucchi L, Limana F, et al. Telomerase expression and activity are coupled with myocyte proliferation and preservation of telomeric length in the failing heart. Proc Natl Acad Sci USA 2001;98:8626-31.

15. Oh $\mathrm{H}, \mathrm{ChiX}$, Bradfute $\mathrm{SB}$, et al. Cardiac muscle plasticity in adult and embryo by heart-derived progenitor cells. Ann N Y Acad Sci 2004;1015:182-9.

16. Oh H, Wang SC, Prahash A, et al. Telomere attrition and Chk2 activation in human heart failure. Proc Natl Acad Sci USA 2003;100:5378-83.

17. Urbanek K, Quaini F, Tasca G, et al. Intense myocyte formation from cardiac stem cells in human cardiac hypertrophy. Proc Natl Acad Sci USA 2003;100:10440-5.

18. Anversa P, Leri A, Kajstura J. Cardiac regeneration. J Am Coll Cardiol 2006;47:1769-76.

19. Hsieh PC, Segers VF, Davis ME, et al. Evidence from a genetic fate-mapping study that stem cells refresh adult mammalian cardiomyocytes after injury. Nat Med 2007;13:970-4.

20. Porrello ER, Mahmoud AI, Simpson E, et al. Transient regenerative potential of the neonatal mouse heart. Science 2011;331:1078-80.

21. Bergmann O, Bhardwaj RD, Bernard S, et al. Evidence for cardiomyocyte renewal in humans. Science 2009;324:98-102.

22. Quaini F, Urbanek K, Beltrami AP, et al. Chimerism of the transplanted heart. N Engl J Med 2002;346:5-15.

23. Oh H, Bradfute SB, Gallardo TD, et al. Cardiac progenitor cells from adult myocardium: homing, differentiation, and fusion after infarction. Proc Natl Acad Sci USA 2003;100:12313-8.

24. Dawn B, Stein AB, Urbanek K, et al. Cardiac stem cells delivered intravascularly traverse the vessel barrier, regenerate infarcted myocardium, and improve cardiac function. Proc Natl Acad Sci USA 2005;102:3766-71.

25. Rota M, Padin-Iruegas ME, Misao Y, et al. Local activation or implantation of cardiac progenitor cells rescues scarred infarcted myocardium improving cardiac function. Circ Res 2008;103:107-16.

26. Bearzi C, Rota M, Hosoda T, et al. Human cardiac stem cells. Proc Natl Acad Sci USA 2007;104:14068-73.

27. Goodell MA, Brose K, Paradis G, Conner AS, Mulligan RC. Isolation and functional properties of murine hematopoietic stem cells that are replicating in vivo. J Exp Med 1996;183:1797-806. 
28. Martin CM, Meeson AP, Robertson SM, et al. Persistent expression of the ATP-binding cassette transporter, Abcg2, identifies cardiac SP cells in the developing and adult heart. Dev Biol 2004;265:262-75.

29. Pfister O, Oikonomopoulos A, Sereti KI, et al. Role of the ATP-binding cassette transporter Abcg2 in the phenotype and function of cardiac side population cells. Circ Res 2008;103:825-35.

30. Pfister O, Mouquet F, Jain M, et al. CD31- but Not CD31+ cardiac side population cells exhibit functional cardiomyogenic differentiation. Circ Res 2005;97:52-61.

31. Wang X, Hu Q, Nakamura Y, et al. The role of the sca-1+/CD31- cardiac progenitor cell population in postinfarction left ventricular remodeling. Stem Cells 2006;24:1779-88.

32. Messina E, De Angelis L, Frati G, et al. Isolation and expansion of adult cardiac stem cells from human and murine heart. Circ Res 2004;95:911-21.

33. Ye J, Boyle A, Shih H, et al. Sca-1+ cardiosphere-derived cells are enriched for Isl1-expressing cardiac precursors and improve cardiac function after myocardial injury. PLoS One 2012;7:e30329.

34. Smith RR, Barile L, Cho HC, et al. Regenerative potential of cardiospherederived cells expanded from percutaneous endomyocardial biopsy specimens. Circulation 2007;115:896-908.

35. Bartosh TJ, Wang Z, Rosales AA, Dimitrijevich SD, Roque RS. 3D-model of adult cardiac stem cells promotes cardiac differentiation and resistance to oxidative stress. J Cell Biochem 2008;105:612-23.

36. Takehara N, Tsutsumi Y, Tateishi K, et al. Controlled delivery of basic fibroblast growth factor promotes human cardiosphere-derived cell engraftment to enhance cardiac repair for chronic myocardial infarction. J Am Coll Cardiol 2008;52:1858-65.

37. Johnston PV, Sasano T, Mills K, et al. Engraftment, differentiation, and functional benefits of autologous cardiosphere-derived cells in porcine ischemic cardiomyopathy. Circulation 2009;120:1075-83, 7 p following 1083.

38. Lee ST, White AJ, Matsushita S, et al. Intramyocardial injection of autologous cardiospheres or cardiosphere-derived cells preserves function and minimizes adverse ventricular remodeling in pigs with heart failure postmyocardial infarction. J Am Coll Cardiol 2011;57:455-65.

39. Pouly J, Bruneval P, Mandet C, et al. Cardiac stem cells in the real world. J Thorac Cardiovasc Surg 2008;135:673-8.

40. Holmes C, Stanford WL. Concise review: stem cell antigen-1: expression, function, and enigma. Stem Cells 2007;25:1339-47.

41. Shenje LT, Field LJ, Pritchard CA, et al. Lineage tracing of cardiac explant derived cells. PLoS ONE 2008;3:e1929.

42. Li Z, Lee A, Huang M, et al. Imaging survival and function of transplanted cardiac resident stem cells. J Am Coll Cardiol 2009;53:1229-40.

43. Andersen DC, Andersen P, Schneider M, Jensen HB, Sheikh SP. Murine "cardiospheres" are not a source of stem cells with cardiomyogenic potential. Stem Cells 2009;27:1571-81.

44. Davis DR, Zhang Y, Smith RR, et al. Validation of the cardiosphere method to culture cardiac progenitor cells from myocardial tissue. PLoS ONE 2009;4:e7195.

45. Kehat I, Kenyagin-Karsenti D, Snir M, et al. Human embryonic stem cells can differentiate into myocytes with structural and functional properties of cardiomyocytes. J Clin Invest 2001;108:407-14.

46. Wong SS, Bernstein HS. Cardiac regeneration using human embryonic stem cells: producing cells for future therapy. Regen Med 2010;5:763-75.

47. Xu XQ, Graichen R, Soo SY, et al. Chemically defined medium supporting cardiomyocyte differentiation of human embryonic stem cells. Differentiation 2008;76:958-70.

48. Xu C, Police S, Rao N, Carpenter MK. Characterization and enrichment of cardiomyocytes derived from human embryonic stem cells. Circ Res 2002;91:501-8.

49. Graichen R, Xu X, Braam SR, et al. Enhanced cardiomyogenesis of human embryonic stem cells by a small molecular inhibitor of p38 MAPK. Differentiation 2008;76:357-70.

50. Gaur M, Ritner C, Sievers R, et al. Timed inhibition of p38MAPK directs accelerated differentiation of human embryonic stem cells into cardiomyocytes. Cytotherapy 2010;12:807-17.

51. Yang L, Soonpaa MH, Adler ED, et al. Human cardiovascular progenitor cells develop from a KDR+ embryonic-stem-cell-derived population. Nature 2008;453:524-8.
52. Zhao Y, Samal E, Srivastava D. Serum response factor regulates a muscle-specific microRNA that targets Hand2 during cardiogenesis. Nature 2005;436:214-20.

53. Zhao Y, Ransom JF, Li A, et al. Dysregulation of cardiogenesis, cardiac conduction, and cell cycle in mice lacking miRNA-1-2. Cell 2007;129: 303-17.

54. Ivey KN, Muth A, Arnold J, et al. MicroRNA regulation of cell lineages in mouse and human embryonic stem cells. Cell Stem Cell 2008;2:219-29.

55. Wong SSY, Ritner C, Aurigui J, et al. miR-125b promotes cardiomyocyte differentiation of human embryonic stem cells. PLoS One 2012, in press.

56. Takeuchi JK, Bruneau BG. Directed transdifferentiation of mouse mesoderm to heart tissue by defined factors. Nature 2009;459:708-11.

57. Huber I, Itzhaki I, Caspi O, et al. Identification and selection of cardiomyocytes during human embryonic stem cell differentiation. FASEB J 2007;21:2551-63.

58. Xu XQ, Zweigerdt R, Soo SY, et al. Highly enriched cardiomyocytes from human embryonic stem cells. Cytotherapy 2008;10:376-89.

59. Kita-Matsuo H, Barcova M, Prigozhina N, et al. Lentiviral vectors and protocols for creation of stable hESC lines for fluorescent tracking and drug resistance selection of cardiomyocytes. PLoS ONE 2009;4:e5046.

60. Ritner C, Wong SS, King FW, et al. An engineered cardiac reporter cell line identifies human embryonic stem cell-derived myocardial precursors. PLoS ONE 2011;6:e16004.

61. Anderson D, Self T, Mellor IR, Goh G, Hill SJ, Denning C. Transgenic enrichment of cardiomyocytes from human embryonic stem cells. Mol Ther 2007;15:2027-36.

62. King FW, Liszewski W, Ritner C, Bernstein HS. High-throughput tracking of pluripotent human embryonic stem cells with dual fluorescence resonance energy transfer molecular beacons. Stem Cells Dev 2011;20:475-84.

63. van Laake LW, Passier R, Monshouwer-Kloots J, et al. Human embryonic stem cell-derived cardiomyocytes survive and mature in the mouse heart and transiently improve function after myocardial infarction. Stem Cell Res 2007;1:9-24.

64. Leor J, Gerecht S, Cohen S, et al. Human embryonic stem cell transplantation to repair the infarcted myocardium. Heart 2007;93:1278-84.

65. Yeghiazarians Y, Gaur M, Zhang Y, et al. Myocardial improvement with human embryonic stem cell-derived cardiomyocytes enriched by p38MAPK inhibition. Cytotherapy 2012;14:223-31.

66. Drukker M, Katchman H, Katz G, et al. Human embryonic stem cells and their differentiated derivatives are less susceptible to immune rejection than adult cells. Stem Cells 2006;24:221-9.

67. Takahashi K, Yamanaka S. Induction of pluripotent stem cells from mouse embryonic and adult fibroblast cultures by defined factors. Cell 2006; 126:663-76.

68. Takahashi $\mathrm{K}$, Tanabe $\mathrm{K}$, Ohnuki $\mathrm{M}$, et al. Induction of pluripotent stem cells from adult human fibroblasts by defined factors. Cell 2007;131:861-72.

69. Zhang J, Wilson GF, Soerens AG, et al. Functional cardiomyocytes derived from human induced pluripotent stem cells. Circ Res 2009;104:e30-41.

70. Zhao T, Zhang ZN, Rong Z, Xu Y. Immunogenicity of induced pluripotent stem cells. Nature 2011;474:212-5.

71. Nakajima F, Tokunaga K, Nakatsuji N. Human leukocyte antigen matching estimations in a hypothetical bank of human embryonic stem cell lines in the Japanese population for use in cell transplantation therapy. Stem Cells 2007;25:983-5.

72. Taylor CJ, Bolton EM, Pocock S, Sharples LD, Pedersen RA, Bradley JA. Banking on human embryonic stem cells: estimating the number of donor cell lines needed for HLA matching. Lancet 2005;366:2019-25.

73. Yabut O, Bernstein HS. The promise of human embryonic stem cells in aging-associated diseases. Aging (Albany NY) 2011;3:494-508.

74. Gore A, Li Z, Fung HL, et al. Somatic coding mutations in human induced pluripotent stem cells. Nature 2011;471:63-7.

75. Mayshar Y, Ben-David U, Lavon N, et al. Identification and classification of chromosomal aberrations in human induced pluripotent stem cells. Cell Stem Cell 2010;7:521-31.

76. Zhou H, Wu S, Joo JY, et al. Generation of induced pluripotent stem cells using recombinant proteins. Cell Stem Cell 2009;4:381-4. 
77. Kim D, Kim CH, Moon JI, et al. Generation of human induced pluripotent stem cells by direct delivery of reprogramming proteins. Cell Stem Cell 2009;4:472-6.

78. Anokye-Danso F, Trivedi CM, Juhr D, et al. Highly efficient miRNA-mediated reprogramming of mouse and human somatic cells to pluripotency. Cell Stem Cell 2011;8:376-88.

79. Okita K, Matsumura Y, Sato Y, et al. A more efficient method to generate integration-free human iPS cells. Nat Methods 2011;8:409-12.

80. Lister R, Pelizzola M, Kida YS, et al. Hotspots of aberrant epigenomic reprogramming in human induced pluripotent stem cells. Nature 2011;471:68-73.

81. Ieda M, Fu JD, Delgado-Olguin P, et al. Direct reprogramming of fibroblasts into functional cardiomyocytes by defined factors. Cell 2010;142:375-86.

82. Zimmermann WH, Melnychenko I, Wasmeier G, et al. Engineered heart tissue grafts improve systolic and diastolic function in infarcted rat hearts. Nat Med 2006;12:452-8.

83. Caspi O, Lesman A, Basevitch Y, et al. Tissue engineering of vascularized cardiac muscle from human embryonic stem cells. Circ Res 2007;100:263-72.

84. Lesman A, Habib M, Caspi O, et al. Transplantation of a tissue-engineered human vascularized cardiac muscle. Tissue Eng Part A 2010;16:115-25.

85. Dvir T, Levy O, Shachar M, Granot Y, Cohen S. Activation of the ERK1/2 cascade via pulsatile interstitial fluid flow promotes cardiac tissue assembly. Tissue Eng 2007;13:2185-93.

86. Brown MA, Iyer RK, Radisic M. Pulsatile perfusion bioreactor for cardiac tissue engineering. Biotechnol Prog 2008;24:907-20.

87. Radisic M, Park H, Shing H, et al. Functional assembly of engineered myocardium by electrical stimulation of cardiac myocytes cultured on scaffolds. Proc Natl Acad Sci USA 2004;101:18129-34.

88. Tandon N, Cannizzaro C, Chao PH, et al. Electrical stimulation systems for cardiac tissue engineering. Nat Protoc 2009;4:155-73.

89. Dvir T, Kedem A, Ruvinov E, et al. Prevascularization of cardiac patch on the omentum improves its therapeutic outcome. Proc Natl Acad Sci USA 2009;106:14990-5.

90. Ott HC, Matthiesen TS, Goh SK, et al. Perfusion-decellularized matrix: using nature's platform to engineer a bioartificial heart. Nat Med 2008;14:213-21.

91. Shimizu T, Yamato M, Isoi Y, et al. Fabrication of pulsatile cardiac tissue grafts using a novel 3-dimensional cell sheet manipulation technique and temperature-responsive cell culture surfaces. Circ Res 2002;90:e40.
92. Furuta A, Miyoshi S, Itabashi Y, et al. Pulsatile cardiac tissue grafts using a novel three-dimensional cell sheet manipulation technique functionally integrates with the host heart, in vivo. Circ Res 2006;98:705-12.

93. Amado LC, Schuleri KH, Saliaris AP, et al. Multimodality noninvasive imaging demonstrates in vivo cardiac regeneration after mesenchymal stem cell therapy. J Am Coll Cardiol 2006;48:2116-24.

94. Sebag IA, Handschumacher MD, Ichinose F, et al. Quantitative assessment of regional myocardial function in mice by tissue Doppler imaging: comparison with hemodynamics and sonomicrometry. Circulation 2005;111:2611-6.

95. Christoforou N, Oskouei BN, Esteso P, et al. Implantation of mouse embryonic stem cell-derived cardiac progenitor cells preserves function of infarcted murine hearts. PLoS ONE 2010;5:e11536.

96. Shake JG, Gruber PJ, Baumgartner WA, et al. Mesenchymal stem cell implantation in a swine myocardial infarct model: engraftment and functional effects. Ann Thorac Surg 2002;73:1919-25; discussion 1926.

97. Abraham MR, Henrikson CA, Tung L, et al. Antiarrhythmic engineering of skeletal myoblasts for cardiac transplantation. Circ Res 2005;97:159-67.

98. Mummery C, Ward-van Oostwaard D, Doevendans P, et al. Differentiation of human embryonic stem cells to cardiomyocytes: role of coculture with visceral endoderm-like cells. Circulation 2003;107:2733-40.

99. Pekkanen-Mattila M, Chapman H, Kerkelä E, et al. Human embryonic stem cell-derived cardiomyocytes: demonstration of a portion of cardiac cells with fairly mature electrical phenotype. Exp Biol Med (Maywood) 2010;235:522-30.

100. Satin J, Kehat I, Caspi O, et al. Mechanism of spontaneous excitability in human embryonic stem cell derived cardiomyocytes. J Physiol (Lond) 2004;559(Pt 2):479-96.

101. Verheule S, Wilson E, Banthia S, et al. Direction-dependent conduction abnormalities in a canine model of atrial fibrillation due to chronic atrial dilatation. Am J Physiol Heart Circ Physiol 2004;287:H634-44.

102. Gepstein L, Ding C, Rahmutula D, et al. In vivo assessment of the electrophysiological integration and arrhythmogenic risk of myocardial cell transplantation strategies. Stem Cells 2010;28:2151-61.

103. Kehat I, Khimovich L, Caspi O, et al. Electromechanical integration of cardiomyocytes derived from human embryonic stem cells. Nat Biotechnol 2004;22:1282-9.

104. Xue T, Cho HC, Akar FG, et al. Functional integration of electrically active cardiac derivatives from genetically engineered human embryonic stem cells with quiescent recipient ventricular cardiomyocytes: insights into the development of cell-based pacemakers. Circulation 2005;111:11-20. 